“ (C) 2019 IEEE. Personal use of this material is permitted. Permission from IEEE must be obtained for all other uses, in any current or future media, including

reprinting/republishing this material for advertising or promotional purposes, creating new collective works, for resale or redistribution to servers or lists, or reuse of any copyrighted component of this work in other works." 


\title{
Interference Management in Underlay In-band D2D-Enhanced Cellular Networks
}

\author{
(Invited Paper) \\ Junnan Yang ${ }^{\ddagger}$, Ming Ding ${ }^{\dagger}$, Guoqiang $\mathrm{Mao}^{\ddagger \dagger}$, Tom Hao Luan* \\ ${ }^{\dagger}$ Data61, CSIRO, Australia \{Email : Ming.Ding@data61.csiro.au\} \\ ${ }^{\ddagger}$ The University of Technology Sydney, Australia \{Email : Junnan.Yang@student.uts.edu.au,g.mao@ieee.org\} \\ *Xidian University, P.R.China \{Email : tom.luan@xidian.edu.cn\}
}

\begin{abstract}
Recently, it has been standardized by the 3rd Generation Partnership Project (3GPP) [1] that deviceto-device (D2D) communications should use uplink resources when coexisting with conventional cellular communications. With uplink resource sharing, both cellular and D2D links cause significant co-channel interference. In this paper, we consider a D2D mode selection criterion based on the maximum received signal strength (MRSS) for each user equipment (UE) to control the D2D-tocellular interference. Specifically, a UE will operate in a cellular mode, if its received signal strength from the strongest base station (BS) is larger than a threshold $\beta$; otherwise, it will operate in a D2D mode. Furthermore, in our study, cellular UEs, D2D transmit UEs and D2D receiver UEs constitute the entire UE set, which is a more practical assumption than dropping more UEs for D2D reception only in existing works. The coverage probability and the area spectral efficiency (ASE) are derived for both the cellular network and the D2D one. Through our theoretical and numerical analyses, we quantify the performance gains brought by D2D communications and provide guidelines for selecting the parameters for network operations.
\end{abstract}

\section{INTRODUCTION}

In the last decade, there has been a sharp increase in the demand for data traffic [2]. To address such massive consumer demand for data communications, especially from the user equipment (UEs) such as smartphones and tablets, many noteworthy technologies have been proposed [3], such as small cell networks (SCNs), cognitive radio, deviceto-device (D2D) communications, etc. In particular, D2D communications allow direct data transfer between a pair of neighboring mobile UEs. Due to the short communication distance between such pairs of D2D UEs, D2D communications hold great promise in improving network performance such as coverage, spectral efficiency, energy efficiency and so on. Recently, it has been standardized by the 3rd Generation Partnership Project (3GPP) [1] that Proximity Services (ProSe) should use uplink resources when coexisting with conventional cellular communications. This means D2D communications will underlay with cellular networks in the uplink. For the underlay inband D2D communications, the most critical issue is to reduce the interference as cellular links and D2D links share the same radio resources.

In parallel with the standardization effort, there has been a surge of academic studies in this area [4-8]. In more detail, using the stochastic geometry theory, Andrews, et al. conducted network performance analyses for the downlink (DL) [9] and the uplink (UL) [10] of SCNs. In which UEs and base stations (BSs) were assumed to be randomly deployed according to a homogeneous Poisson distribution. In [11], Peng developed an analytical framework for the D2D underlaid cellular network in the DL, where the Rician fading channel model was adopted to model the smallscale fast fading for the D2D communication links. In [4], Liu provided a unified framework to analyze the downlink outage probability in a multi-channel environment with Rayleigh fading, where D2D UEs were selected based on the average received signal strength from the nearest BS, which is equivalent to a distance-based selection. In [12], George proposed exclusion regions to protect cellular receivers from excessive interference from active D2D transmitters.

In this paper, we propose a more generalized framework which takes into account a novel interference management scheme which based on the maximum received signal strength of UEs from BSs and shed new insight on the interference management of coexistent D2D and cellular transmissions. The main contributions of this paper are summarized as follows:

- We study a practical network in which UEs can adaptively switch between conventional cellular UEs and D2D UEs. In most previous studies, the authors have considered D2D receiver UEs as an additional tier of nodes, independent of the cellular UEs and the D2D transmitter UEs. Such tier of D2D receiver UEs without cellular capabilities appears from nowhere and is hard to justify in practice. In our study, cellular UEs, D2D transmit UEs and D2D receiver UEs constitute the entire UE set, which is a more practical assumption than dropping more UEs for D2D reception only. Moreover, our framework considers interference management, the power control of cellular UEs and shadow fading, which are partly ignored in the existing studies.

- With uplink resource sharing, both cellular and D2D links cause significant co-channel interference. Hence, we proposed a tractable interference management scheme for each user equipment (UE) to control the cochannel interference. Specifically, a UE will operate in a cellular mode if its received signal strength from the strongest base station (BS) is larger than a threshold; 
otherwise, it will operate in a D2D mode. Such maximum received signal strength based mode selection scheme is more practical than the distance-based mode selection in most existing studies because in practice it is possible that the strongest received signal strength is not associated with the closest BS but the one with the minimum path loss.

- Our results demonstrate that the D2D links can provide a considerable the area spectral efficiency (ASE) gain when the threshold parameter is appropriately chosen.

The rest of this paper is organized as follows. In Section II, we introduce the system model and assumptions used in this paper. Section III presents our main results. We provide numerical results and more discussion in Section IV and conclude our work in Section V.

\section{System Model}

In this section, we present the system model and assumptions that are used in this paper.

\section{A. The Path Loss Model}

We consider a D2D underlaid UL cellular network, and we assume the BSs are spatially distributed according to a homogeneous Poisson point process (PPP) $\Phi_{b}$ of intensity $\lambda_{b}$, i.e., $\Phi_{b}=\left\{X_{i}\right\}$, where $X_{i}$ denotes the spatial locations of the $i$ th BS. Moreover, the UEs are also distributed in the network region according to another independent homogeneous PPP $\Phi_{u}$ of intensity $\lambda_{u}$, each UE choose operate mode based on the downlink received signal power from BSs at the first time slot.

We focus on the typical receiver at the origin. The received power for a typical UE from a BS $b$ can be written as

$$
P_{b}^{\mathrm{rx}}=A_{B} P_{B} \mathcal{H}_{\mathrm{B}}(b) R^{-\alpha_{B}},
$$

where $A_{B}=10^{\frac{1}{10}} A_{B}^{\mathrm{dB}}$ is a constant determined by the transmission frequency for BS-to-UE links, $P_{B}$ is the transmission power of a $\mathrm{BS}$ which is constant, $\mathcal{H}_{\mathrm{B}}(b)$ is the lognormal shadowing from the BS $b$ to the typical UE.

There are two modes for UEs in the considered D2Denabled UL cellular network, i.e., cellular mode and D2D mode. Each UE is assigned with a mode to operate according to the comparison of the received DL power from its serving BS with a threshold. In more detail,

$$
\text { Mode }=\left\{\begin{array}{ll}
\text { Cellular, } & \text { if } P^{*}=\max _{b}\left\{P_{b}^{\text {rx }}\right\}>\beta \\
\text { D2D, } & \text { otherwise }
\end{array},\right.
$$

where the string variable Mode takes the value of 'Cellular' or 'D2D'. In particular, for a tagged UE, if $P^{*}$ is large than a specific threshold $\beta>0$, then the UE is not appropriate to work in the $\mathrm{D} 2 \mathrm{D}$ mode due to its potentially large interference, and hence it should operate in the cellular mode and directly connect with a BS. Otherwise, it should operate in the D2D mode. The UEs that are associated with cellular BSs are referred to as cellular UEs (CU) and the distance from a $\mathrm{CU}$ to its associated $\mathrm{BS}$ is denoted by $R^{B}$. For a D2D UE, we adopt the same assumption in [4] that it randomly decides to be a $\mathrm{D} 2 \mathrm{D}$ transmitter or $\mathrm{D} 2 \mathrm{D}$ receiver with equal probability at the beginning of each time slot, and a D2D receiver UE selects the strongest D2D transmitter $\mathrm{UE}$ for signal reception.
The path loss functions for the UE-to-BS links and UEto-UE links can be captured as following

$$
P L_{\text {cellular }}^{\mathrm{dB}}=A_{B}^{\mathrm{dB}}+\alpha_{B} 10 \log _{10} R+\xi_{B}
$$

and

$$
P L_{\mathrm{D} 2 \mathrm{D}}^{\mathrm{dB}}=A_{D}^{\mathrm{dB}}+\alpha_{D} 10 \log _{10} R+\xi_{D}
$$

where the path loss is expressed in $\mathrm{dB}$ unit, $A_{B}^{\mathrm{dB}}$ and $A_{D}^{\mathrm{dB}}$ are constants determined by the transmission frequency, $\alpha_{B}$ and $\alpha_{D}$ are path loss exponents for the UE-to-BS links and UE-to-UE links. Moreover, we denote by $\mathcal{H}_{\mathrm{B}}$ and $\mathcal{H}_{\mathrm{D}}$ the lognormal fading coefficients of a CU-to-BS link and a UE-to-UE link, and we assume that $\mathcal{H}_{\mathrm{B}}=\exp \left(\kappa \xi_{\mathrm{db}}^{\mathrm{B}}\right)$ and $\mathcal{H}_{\mathrm{D}}=\exp \left(\kappa \xi_{\mathrm{db}}^{\mathrm{D}}\right)$ are lognormal fading, where $\kappa=$ $-\operatorname{In} 10 / 10$ is a constant, i.e., $\xi_{d b}^{B} \sim \mathcal{N}\left(0, \sigma_{B}^{2}\right)$ and $\xi_{\mathrm{db}}^{\mathrm{D}} \sim$ $\mathcal{N}\left(0, \sigma_{D}^{2}\right)$

Base on the above system model, we can obtain the intensity of $\mathrm{CU} \lambda_{c}$ as $\lambda_{c}=q \lambda_{u}$, where $q$ denotes the probability of $P^{*}>\beta$ and will be derived in closed-form expressions in Section III. It is apparent that the D2D UEs are distributed following another non-homogenous PPP $\Phi_{d}$, the intensity of D2D UE $\lambda_{d}$ is $\lambda_{d}=(1-q) \lambda_{u}$.

\section{B. The Scenario Description}

We assume an underlaid D2D model. That is, each D2D transmitter reuses the frequency with cellular UEs, which incurs inter-tier interference from the D2D tier to the cellular tier. However, there is no intra-cell interference between cellular UEs since we assume an orthogonal multiple access technique in a BS. It follows that there is only one uplink transmitter in each cellular BS. Here, we consider a fully loaded network with $\lambda_{u} \gg \lambda_{b}$, so that on each timefrequency resource block, each BS has at least one active UE to serve in its coverage area. Note that the case of $\lambda_{u}<\lambda_{b}$ is not trivial, which even changes the capacity scaling law [13]. Due to the page limit, we leave the study of $\lambda_{u}<\lambda_{b}$ as our future work.

Moreover, we assume a channel inversion strategy for the power control for cellular UEs, i.e.,

$$
P_{c_{i}}=P_{0}\left(\frac{\mathrm{R}_{\mathrm{i}}^{\alpha_{\mathrm{B}}}}{\mathcal{H}_{\mathrm{c}_{\mathrm{i}}} A_{\mathrm{B}}}\right)^{\varepsilon},
$$

where $A_{B}=10^{\frac{1}{10}} A_{B}^{\mathrm{dB}}$ is a constant determined by the transmission frequency for BS-to-UE links, $P_{c_{i}}$ is the transmission power of the $i$-th cellular UE, $R_{i}$ is the distance of the $i$-th link from a $\mathrm{CU}$ to the target $\mathrm{BS}, \alpha_{B}$ denotes the pathloss exponent, $\epsilon \in(0,1]$ is the fractional path loss compensation, $P_{0}$ is the receiver sensitivity. For $\mathrm{BS}$ and D2D transmitters, they use constant transmit powers $P_{B}$ and $P_{d}$, respectively. $\mathcal{H}_{\mathrm{c}_{\mathrm{i}}}$ is the lognormal shadowing of the $i$-th cellular link.

According to [9], the coverage probability of the typical receiver which is located in the origin is defined as

$$
P_{\text {Mode }}\left(T, \lambda_{\text {Mode }}\right)=\operatorname{Pr}[\operatorname{SINR}>T],
$$

where $T$ is the signal-to-interference-plus-noise ratio (SINR) threshold, the subscript string variable Mode takes the value of 'Cellular' or 'D2D'. 


\section{MAIN RESULTS}

\section{A. The Probability of UE Operating in the Cellular Mode}

In this subsection, we present our results on the probability that a UE operates in cellular mode and the equivalence distance distributions in cellular mode and D2D mode respectively. The derived results will be used in the analysis of the coverage probability later.

Lemma 1. The probability that a generic mobile UE registers to the strongest BS and operates in cellular mode is given by

$$
q=1-\exp \left(-\pi \lambda_{B}\left(\frac{A_{B} P_{B}}{\beta}\right)^{2 / \alpha_{B}} \cdot e^{\frac{2 \sigma_{B}^{2}}{\alpha_{B}}}\right)
$$

\section{Proof: See Appendix A.}

Note that Eq.(7) explicitly account for the effects of channel fading, path loss, transmit power, the spatial distribution of BSs and the RSS threshold $\beta$. The probability that the UE operates in D2D mode is $(1-q)$. From the result, one can see that the PPP $\phi_{u}$ can be divided into two PPPs: the PPP with intensity $q \lambda_{u}$ and the PPP with intensity $(1-q) \lambda_{u}$, which consist of cellular UEs and D2D UEs, respectively. We assume these two PPP are independent.

B. Equivalence Distance Distributions of the D2D tier and the cellular tier

The distance $R_{i}^{B}$ from a typical user to its associated BS (maximum downlink receive power including lognormal fading) is an important quantity to calculate the average power. In this subsection, we derived the pdf of $\bar{R}_{i}$, and then we derived the distribution of the distance of D2D links. We can also derive the average transmission power of CUs using this equivalence theorem and a simple validation is showed in this subsection.

Lemma 2. The probability density function (pdf) of $\bar{R}_{i}$ can be written as

$$
f_{\overline{R_{i}}}(r)=\frac{2 \pi \lambda_{B} r \cdot \exp \left(-\pi \lambda_{B} r^{2} \cdot e^{\frac{2 \sigma_{B}^{2}}{\alpha_{B}^{2}}}+\frac{2 \sigma_{B}^{2}}{\alpha_{B}^{2}}\right)}{1-\exp \left(-\pi \lambda_{B}\left(\frac{B_{B}}{\beta}\right) 2 / \alpha_{B} \cdot e^{\frac{2 \sigma^{2}}{\alpha_{B}^{2}}}\right)},
$$

where $B_{B}=A_{B} P_{B}$ is a constant.

Proof: The probability density function (PDF) of $\overline{R_{i}}$ can be derived using the simple fact that the null probability of a 2-D Poisson process in area $\mathrm{A}$ is $\exp (-\lambda A)$, and we have known that $\bar{R}_{i} \leq\left(\frac{\beta}{B_{B}}\right)^{-1 / \alpha_{B}}$, which leads to Lemma 2 .

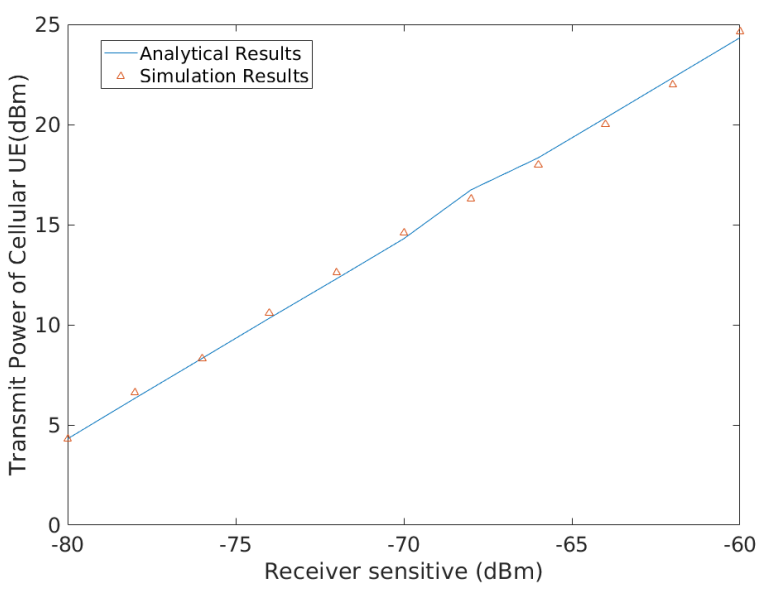

Figure 1. The transmit power of cellular UEs varies with $p_{0}$.

As a numerical example, we plot cellular users' transmit power in Fig. 1. The analytical result is derived from Eq.(??) and Eq.(8), which valid our analysis. It shows that the analytical result matched well with the numerical result, which validates our analysis.

Lemma 3. The typical D2D transmitter selects the equivalent nearest $U E$ as a potential receiver, if the potential $D 2 D$ receiver is operating in a cellular mode, D2D TU must search for another receiver. We approximate the second neighbor as the receiver under this situation. The approximate cumulative distribution function $(C D F)$ of $\bar{R}_{d}$ can be written as

$$
\begin{aligned}
\operatorname{Pr}\left[\bar{R}_{d}<R\right] & \\
& \approx \int_{R+t}^{\infty}\left(\int_{0}^{R} f_{R_{d}}\left(\bar{R}_{d}\right) d \bar{R}_{d}\right) f_{R_{d}}\left(r_{1}\right) d r_{1} \\
& +\int_{t}^{R+t}\left(\int_{0}^{r_{1}-t} f_{R_{d}}\left(\bar{R}_{d}\right) d \bar{R}_{d}\right. \\
& +\int_{r_{1}-t}^{R}(1-P c) \cdot f_{R_{d}}\left(\bar{R}_{d}\right) d \bar{R}_{d} \\
& \left.+\int_{r_{1}-t}^{R} P c \cdot f_{R_{d_{2}}}\left(\bar{R}_{d}\right) d \bar{R}_{d}\right) f_{R_{d}}\left(r_{1}\right) d r_{1},
\end{aligned}
$$

where $r_{1}$ is the equivalent distance from $T U$ to the strongest $B S, t=\left(\frac{\beta}{B_{B}}\right)^{-1 / \alpha_{B}} . P c$ is the probability of a D2D receiver be a $C U$.

Proof: See Appendix B.

\section{The Coverage Probability}

Consider an arbitrary BS in cellular mode or UE in D2D mode. The SINR experenced at the receiver can be located in an arbitrary location and can be written as

$$
S I N R=\frac{S}{\sum_{x_{c_{i}} \in \phi_{c}} B_{i}^{B} \mathcal{H}_{\mathrm{i}}^{\mathrm{B}} R_{B, i}^{-\alpha}{ }^{-\alpha}+\sum_{X_{d_{j}} \in \phi_{d}} B_{j}^{D} \mathcal{H}_{\mathrm{j}}^{\mathrm{D}} R_{D, j}^{-\alpha}+\eta_{c, d}},
$$

where $B_{i}^{B}=P_{C}^{i} \cdot A_{B}$ and $B^{D}=P_{D} \cdot A_{D}$ are constants which based on transmission power of the $i$ th $C U$ and the TUs, $A_{D}=10^{\frac{1}{10}} A_{D}^{\mathrm{dB}}, \mathcal{H}_{\mathrm{i}}^{\mathrm{B}}$ and $\mathcal{H}_{\mathrm{j}}^{\mathrm{D}}$ are the lognorm fading in $i$ th cellular uplink link and $j$ th D2D link, $R_{B, i}$ and $R_{D, i}$ 
are the distance from the $i$ th $\mathrm{CU}$ and $j$ th TU to the typical receiver. The Equivalence distance $\bar{R}_{B i}=\mathcal{H}_{\mathrm{B}, \mathrm{i}}^{-1 / \alpha_{\mathrm{B}}} \mathrm{R}_{\mathrm{B}, \mathrm{i}}$ and $\bar{R}_{D}=\mathcal{H}_{\mathrm{D}, \mathrm{j}}^{-1 / \alpha_{\mathrm{D}}} \mathrm{R}_{\mathrm{D}, \mathrm{j}}, \alpha_{B}$ and $\alpha_{D}$ are path-loss exponent for cellular links and D2D links, respectively. $\eta_{c, d}$ is the noise for $\mathrm{BS}$ or receive $\mathrm{UE}$.

1) Cellular mode: Let us consider a typical uplink, as the underlying PPP is stationary, without loss of generality we assume that the typical receiver is located at the origin. This analysis indicates the spatially averaged performance of the network by Slivnyak's theorem [9] of PPP. Henceforth, we only need to focus on characterizing the performance of a typical link.

Lemma 4. The complementary cumulative distribution function $(C C D F)$ of the SINR at a typical BS (located in the origin)

$$
\operatorname{Pr}[\mathrm{SINR}>\mathrm{T}]
$$

$$
=\int_{0}^{\mathrm{t}} \int_{\omega=-\infty}^{\infty}\left[\frac{e^{i \omega / T}-1}{2 \pi i \omega}\right] \mathcal{F}_{S I N R^{-1}}(\omega) d \omega f_{\bar{R}_{i}}(r) d r,
$$

where $\mathcal{F}_{S I N R^{-1}}(\omega)$ denotes the conditional characteristic function of $\frac{1}{S I N R}$.

$$
\begin{array}{r}
\mathcal{F}_{S I N R^{-1}}(\omega) \\
=\exp \left\{-2 \pi \lambda_{B} e^{\frac{2 \sigma^{2}}{\alpha_{B}^{2}}} \int_{t}^{\infty}\left(1-\int_{0}^{t} \exp (-1 \times\right.\right. \\
\left.\left.\left.\frac{i \omega}{\left(\overline{R_{B, 0}}\right)^{\alpha_{B}(\varepsilon-1)}} r^{\alpha_{B} \varepsilon}(\tau)^{-\alpha_{B}}\right) f_{\overline{R_{i}}}(r) d r\right) \tau d \tau\right\} \\
\times \exp \left\{-\pi(1-q) \lambda_{u} e^{\frac{2 \sigma^{2}}{\alpha_{B}^{2}}} \int_{t}^{\infty}(1-\exp (-1 \times\right. \\
\left.\left.\frac{i \omega A_{B}^{\varepsilon} P_{d}}{P_{0} \cdot\left(\overline{R_{B, 0}}\right)^{\alpha_{B}(\varepsilon-1)}}(L)^{-\alpha_{B}}\right) L d L\right\} \\
\times \exp \left(-\frac{P_{0}}{\overline{\left(\mathrm{A}_{\mathrm{B}}\right)^{\varepsilon-1}}} \cdot\left(\overline{R_{B, 0}}\right)^{\alpha_{B}(\varepsilon-1)}\right) .
\end{array}
$$

Proof: See Appendix C.

2) Coverage Probability of D2D Mode: From [4], one can see that in order to derive the coverage probility of a generic D2D UE, we only need to derive the coverage probaility for a typical D2D UE receiving signal. Similar to the analysis in section III-C1, we focus on a typical D2D UE which is located at the origin $o$ and scheduled to receive data from another D2D UE. Following Slivnyak's theorem for PPP, the coverage probability result derived for the typical D2D UE holds also for any generic D2D UE located at any location. We use the result in Lemma 3 as the distance distribution of D2D links, then we have Lemma 5.

Lemma 5. The CCDF of the SINR at a typical D2D UE (located in the origin)

$\operatorname{Pr}[\operatorname{SINR}>T]$

$$
=\int_{0}^{\infty} \int_{\omega=-\infty}^{\infty}\left[\frac{e^{i \omega / T}-1}{2 \pi i \omega}\right] \mathcal{F}_{S I N R^{-1}}(\omega) d \omega f_{\overline{R_{d}}}(r) d r,
$$

where $\mathcal{F}_{S I N R^{-1}}(\omega)$ denotes the conditional characteristic function of $\frac{1}{S I N R}$.

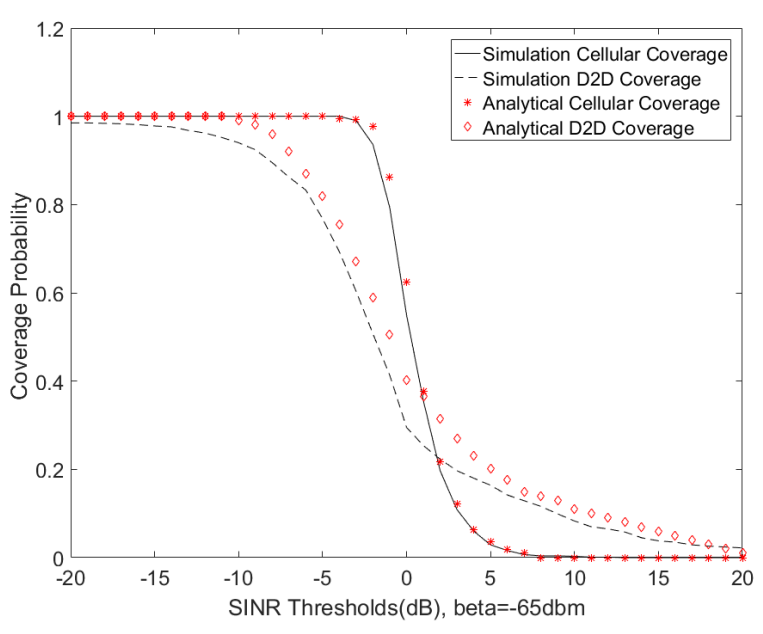

Figure 2. The coverage probability performance.

$$
\begin{aligned}
& \mathcal{F}_{S I N R^{-1}}(\omega)=\exp \left\{-2 \pi \lambda_{B} e^{\frac{2 \sigma^{2}}{\alpha_{B}^{2}}} \int_{0}^{\infty}\left(1-\int_{0}^{t} \exp (-1 \times\right.\right. \\
&\left.\left.\left.\frac{i \omega}{P_{d}\left(\overline{R_{d, 0}}\right)^{-\alpha_{d}}} P_{0} A_{B}^{-\varepsilon} r^{\alpha_{B} \varepsilon}(\tau)^{-\alpha_{B}}\right) f_{\overline{R_{i}}}(r) d r\right) \tau d \tau\right\} \\
& \quad \times \exp \left\{-\pi(1-q) \lambda_{u} e^{\frac{2 \sigma_{d}^{2}}{\alpha_{d}^{2}}} \int \frac{\infty}{R_{d, 0}}(1-\exp (-1 \times\right. \\
&\left.\left.\frac{i \omega}{\left(\overline{R_{d, 0}}\right)^{-\alpha_{d}}}(L)^{-\alpha_{B}}\right) L d L\right\} \exp \left(-\frac{i \omega \eta_{d}}{P_{d} A_{D}\left(\overline{R_{d, 0}}\right)^{-\alpha_{d}}}\right)
\end{aligned}
$$

and

$$
f_{\overline{R_{d}}}(r)=\frac{\partial \operatorname{Pr}\left[\bar{R}_{d}>R\right]}{\partial \bar{R}_{d}} .
$$

Proof: The proof is very similar to that for the cellular mode, and hence we can see Appendix C.

\section{Simulations AND Discussion}

In this section, we use numerical results to validate our results on the performance of the considered D2D-enabled UL cellular network. According to the 3GPP LTE specifications [14], we set the BS intensity to $\lambda_{b}=5 \mathrm{BSs} / \mathrm{km}^{2}$, which results in an average inter-site distance of about $500 \mathrm{~m}$. The UE intensity is chosen as $\lambda_{u}=300 \mathrm{UEs} / \mathrm{km}^{2}$, which is a typical value in $5 \mathrm{G}$ [15]. The transmit power of each BS and each D2D transmitter are set to $P_{B}=46 \mathrm{dBm}$ and $10 \mathrm{dBm}$, respectively. Moreover, the path-loss exponents are set to $\alpha_{c}=\alpha_{d}=3.75$, and the path-loss constants are $A_{B}=10^{-3.29}, A_{D}=10^{-5.578}$. The threshold for selecting cellular mode communication is $\beta=-65 \mathrm{dBm}$. The lognormal shadowing standard deviation is $8 \mathrm{~dB}$ between UEs to BSs and $7 \mathrm{~dB}$ between UEs to UEs. The noise powers are set to $-95 \mathrm{dBm}$ for a UE receiver and $-114 \mathrm{dBm}$ for a $\mathrm{BS}$ receiver, respectively.

\section{A. The Results on the Coverage Probability}

In Fig.2, we plot the coverage probability for both a typical cellular UE and a typical D2D UE. From this figure, we can draw the following observations: 


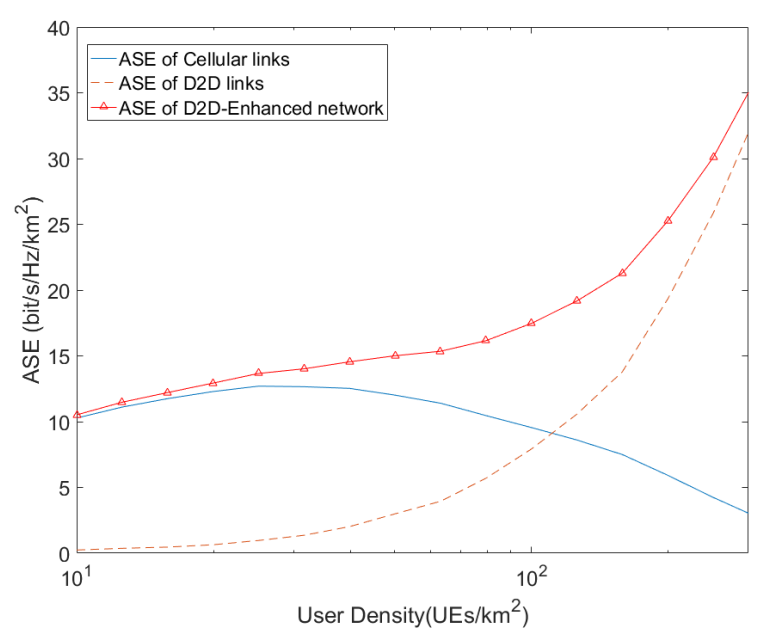

Figure 3. The ASE performance with various densities of UEs.

- Our analytical results match well with the simulation results, which validates our analysis and shows that the adopted model accurately captures the features of D2D communications.

- The coverage probability decreases with the increase of SINR threshold because a higher SINR requirement implies more difficulty in satisfying the coverage criterion in Eq.(6).

- In the D2D mode, the analytical results are shown to be larger than the simulation results. This is because we approximate the distance from a typical D2D TU to a typical D2D RU as that from a second nearest D2D UE to such typical D2D RU, when the nearest D2D UE to such typical D2D RU selects the cellular mode. However, the real distance from a typical D2D TU to a typical D2D RU could be larger than the approximate distance used in our analysis addressed in section III-B.

\section{B. The Results on the ASE}

In Fig.3, we display the ASE results with $\gamma_{0}=0 \mathrm{~dB}$. Since $A^{\mathrm{ASE}}\left(\lambda_{b}, \lambda_{u}, \gamma_{0}\right)$ is a function of the coverage probability, which has been validated in Fig.2, we only show analytical results in Fig.3. From Fig.3, we can draw the following observations:

- The total ASE increases with the increase of the intensity of UE. This is because the spectral reuse factor increases with the number of UEs in the network.

- When the intensity of UE is around $\lambda=100 \mathrm{UEs} / \mathrm{km}^{2}$, the enabled-D2D links have a comparable contribution to the total ASE as the cellular links. This is because there are around 1/3 UEs operating in cellular mode so that the density of cellular links and D2D links are similar, and base on the coverage probability in D2D tier and cellular tier which are operating above a given threshold $(\mathrm{SINR}>0 \mathrm{~dB})$, we can see almost same proportion in this two modes. Hence they make roughly equal contributions to the ASE performance.

- When the network is dense enough, i.e., $\lambda_{u} \in$ $[50,250] \mathrm{UEs} / \mathrm{km}^{2}$, which is the practical range of the UE intensity for the existing $4 \mathrm{G}$ network and the future $5 \mathrm{G}$ network [3], the total ASE performance increases

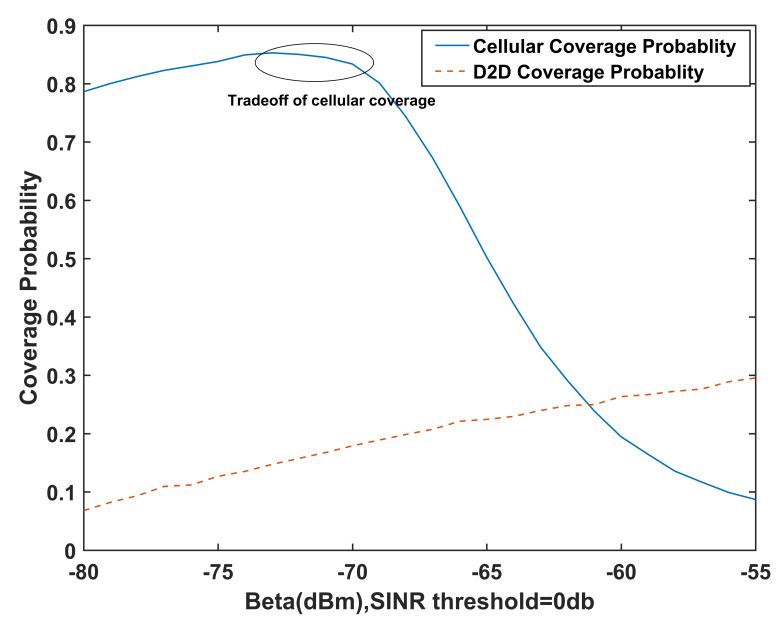

Figure 4. The coverage probability performance with various values of $\beta$.

quickly, while the ASE of the cellular network stays on top of $5 \mathrm{bps} / \mathrm{Hz} / \mathrm{km}^{2}$, providing a decent and ubiquitous coverage.

\section{The Performance Impact of $\beta$ on the ASE}

In this subsection, we investigate the performance impact of $\beta$ on the coverage probability on both the cellular tier and the D2D tier, which is shown in Fig.4. From this figure, we can see that the coverage probability performance of the cellular mode is a concave function, and the optimal beta in this scenario is around $-72 \mathrm{dBm}$, which achieves the best coverage probability performance for cellular users. This means that with a proper choice of $\beta$, enabling D2D communications not only improves the ASE of the network, but also benefits the coverage of cellular users. This is because the cell edge UEs in the conventional UL cellular network will be offloaded to D2D modes to enjoy a better coverage performance.

\section{CONCLUSION}

In this paper, we proposed an interference management method in a D2D enhanced uplink cellular network, where the location of the mobile UEs and the BSs are modeled as PPP. This mode selection method mitigates large interference from D2D transmitter to the cellular network. Using a stochastic geometric approach, we analytically evaluated the coverage probability and the ASE for various values of the mode selection threshold $\beta$. Our results showed that enabling D2D communications in cellular networks can improve the total ASE while having a minor performance impact on the cellular network.

\section{Appendix A: Proof of LEMma 1}

The probability of the RSS large than the threshold is given by

$$
P=\operatorname{Pr}\left[\max \left(A_{B} P_{B} \mathcal{H}_{\mathrm{B}} R^{-\alpha_{B}}\right)>\beta\right],
$$

where we use the standard power loss propagation model with path loss exponent $\alpha_{B}$ (for UE-BS links) and $\alpha_{D}$ (for 
UE-UE links). The the probability that a generic mobile UE operates in cellular mode is

$$
\begin{aligned}
q & =1-\operatorname{Pr}\left[\max \left(A_{B} P_{B} \mathcal{H}_{\mathrm{B}} R^{-\alpha_{B}}\right) \leq \beta\right] \\
& =1-\exp \left(-\Lambda\left(\left[0,\left(\frac{\beta}{A_{B} P_{B}}\right)^{-1 / \alpha_{B}}\right]\right)\right) \\
& =1-\exp \left(-\pi \lambda_{B}\left(\frac{A_{B} P_{B}}{\beta}\right)^{2 / \alpha_{B}} \cdot e^{\frac{2 \sigma^{2}}{\alpha_{B}^{2}}}\right)
\end{aligned}
$$

which concludes our proof.

\section{APPENDIX B: PROOF OF LEMMA 3}

If there is no difference between CUs and D2D UEs, the pdf of the distance between UEs is

$$
f_{R_{d}}(r)=2 \pi \lambda_{t u} r \cdot \exp \left[-\pi \lambda_{t u} r^{2} \cdot e^{\frac{2 \sigma_{D}^{2}}{\alpha_{D}^{2}}}+\frac{2 \sigma_{D}^{2}}{\alpha_{D}^{2}}\right] .
$$

According to [16], the second neighbor point is distributed as

$$
f_{R_{d_{2}}}(r)=2 \pi^{2} \lambda_{t u}^{2} r^{3} \cdot \exp \left[-\pi \lambda_{t u} r^{2} \cdot e^{\frac{2 \sigma_{D}^{2}}{\alpha_{D}^{2}}}+\frac{4 \sigma_{D}^{2}}{\alpha_{D}^{2}}\right]
$$

where $P c$ is the probability of the potential D2D receiver operating in cellular mode, and it can be calculated as

$$
P c=\arccos \left(\frac{\bar{R}_{d}+r_{1}^{2}-t^{2}}{2 \bar{R}_{d} r_{1}}\right) / \pi,
$$

which concludes our proof.

\section{ApPEndix C: Proof of LEMMA 4}

Condition on the strongest $\mathrm{BS}$ being at a distance $R_{B, 0}$ from the typical $\mathrm{CU}$, the Equivalence distance $\overline{R_{B, 0}}=$ $\mathcal{H}_{\mathrm{B}}^{-1 / \alpha_{\mathrm{B}}} \mathrm{R}_{\mathrm{B}, 0}\left(\overline{R_{B, 0}} \leq\left(\frac{\beta}{B_{B}}\right)^{-1 / \alpha_{B}}\right)$, probability of coverage averaged over the plane is

$$
\begin{aligned}
p_{c}(T, \lambda) & =\operatorname{Pr}[S I N R>T] \\
& =\operatorname{Pr}\left[\frac{1}{S I N R}<\frac{1}{T}\right] \\
& =\int_{0}^{\mathrm{t}} \operatorname{Pr}\left[\frac{1}{S I N R}<\frac{1}{T} \mid \overline{R_{B, 0}}\right] f_{\overline{R_{i}}}(r) d r
\end{aligned}
$$

where $i=\sqrt{-1}$ is the imaginary unit; The inner intergral is the conditional PDF of $\frac{1}{S I N R} ; \mathcal{F}_{S I N R^{-1}}(\omega)$ denotes the conditional characteristic function of $\frac{1}{S I N R}$ which can be written by

$$
\begin{aligned}
\mathcal{F}_{S I N R^{-1}}(\omega) & \\
= & \mathbb{E}_{\phi}\left[\exp \left(-i \omega \frac{1}{S I N R}\right) \mid \overline{R_{B, 0}}\right] \\
= & E_{\phi_{c}}\left[\exp \left(-\frac{i \omega}{\frac{P_{0}}{\left(\mathrm{~A}_{\mathrm{B}}\right)^{\varepsilon-1}} \cdot\left(\overline{R_{B, 0}}\right)^{\alpha_{B}(\varepsilon-1)}}\left(I_{C}\right)\right)\right] \\
& \times \mathbb{E}_{\phi_{d}}\left[\exp \left(-\frac{i \omega}{\frac{P_{0}}{\left(\mathrm{~A}_{\mathrm{B}}\right)^{\varepsilon-1}} \cdot\left(\overline{R_{B, 0}}\right)^{\alpha_{B}(\varepsilon-1)}}\left(I_{D}\right)\right)\right] \\
& \times \exp \left(-\frac{i \omega \eta}{\frac{P_{0}}{\left(\mathrm{~A}_{\mathrm{B}}\right)^{\varepsilon-1}} \cdot\left(\overline{R_{B, 0}}\right)^{\alpha_{B}(\varepsilon-1)}}\right),
\end{aligned}
$$

and using the definition of the Laplace transform yields from [9], we have

$$
\begin{aligned}
\mathcal{L}_{\mathrm{I}_{\mathrm{c}}}(s)= & \mathbb{E}_{\phi_{c}}\left[\exp \left(-s I_{c}\right)\right] \\
= & \exp \left\{-2 \pi \lambda_{B} e^{\frac{2 \sigma^{2}}{\alpha_{B}^{2}}} \int_{\overline{R_{B, 0}}}^{\infty}\left(1-\int_{0}^{t} \exp (-1 \times\right.\right. \\
& \left.\left.\left.s P_{0} A_{B}^{(1-\varepsilon)} r^{\alpha_{B} \varepsilon}(\tau)^{-\alpha_{B}}\right) f_{\overline{R_{i}}}(r) d r\right) \tau d \tau\right\} .
\end{aligned}
$$

Plugging in $s=\frac{i \omega}{\frac{P_{0}}{\left(\mathrm{~A}_{\mathrm{B}}\right)^{\varepsilon-1}} \cdot\left(\overline{R_{B, 0}}\right)^{\alpha} B^{(\varepsilon-1)}}$ gives

$$
\begin{aligned}
& \mathbb{E}_{\phi_{c}}\left[\exp \left(-\frac{i \omega}{\frac{P_{0}}{\left(\mathrm{~A}_{\mathrm{B}}\right)^{\varepsilon-1}} \cdot\left(\overline{R_{B, 0}}\right)^{\alpha_{B}(\varepsilon-1)}}\left(I_{C}\right)\right)\right. \\
& =\exp \left\{-2 \pi \lambda_{B} e^{\frac{2 \sigma^{2}}{\alpha_{B}^{2}}} \int_{t}^{\infty}\left(1-\int_{0}^{t} \exp (-1 \times\right.\right. \\
& \left.\left.\left.\frac{i \omega}{\left(\overline{R_{B, 0}}\right)^{\alpha_{B}(\varepsilon-1)}} r^{\alpha_{B} \varepsilon}(\tau)^{-\alpha_{B}}\right) f_{\overline{R_{i}}}(r) d r\right) \tau d \tau\right\}
\end{aligned}
$$

which concludes our proof.

\section{REFERENCES}

[1] J.S.Roessler, "LTE-Advanced (3GPP Rel.12) technology introduction white paper," 2015

[2] C. V. N. Index, "Global mobile data traffic forecast update, 20152020 white paper," link: http://goo. gl/ylTuVx, 2016.

[3] D. Lopez-Perez, M. Ding, H. Claussen, and A. H. Jafari, "Towards $1 \mathrm{Gbps} / \mathrm{UE}$ in cellular systems: Understanding ultra-dense small cell deployments," IEEE Communications Surveys Tutorials, vol. 17, no. 4, pp. 2078-2101, Fourthquarter 2015.

[4] J. Liu, H. Nishiyama, N. Kato, and J. Guo, "On the outage probability of device-to-device-communication-enabled multichannel cellular networks: An RSS-Threshold-Based perspective," IEEE Journal on Selected Areas in Communications, vol. 34, no. 1, pp. 163-175, Jan 2016.

[5] H. ElSawy, E. Hossain, and M. S. Alouini, "Analytical modeling of mode selection and power control for underlay D2D communication in cellular networks," IEEE Transactions on Communications, vol. 62, no. 11, pp. 4147-4161, Nov 2014.

[6] N. Lee, X. Lin, J. G. Andrews, and R. W. Heath, "Power control for D2D underlaid cellular networks: Modeling, algorithms, and analysis," IEEE Journal on Selected Areas in Communications, vol. 33, no. 1 , pp. 1-13, 2015.

[7] X. Lin, J. G. Andrews, and A. Ghosh, "Spectrum sharing for deviceto-device communication in cellular networks," IEEE Transactions on Wireless Communications, vol. 13, no. 12, pp. 6727-6740, Dec 2014.

[8] K. S. Ali, H. ElSawy, and M. S. Alouini, "Modeling cellular networks with full-duplex d2d communication: A stochastic geometry approach," IEEE Transactions on Communications, vol. 64, no. 10, pp. 4409-4424, Oct 2016.

[9] J. G. Andrews, F. Baccelli, and R. K. Ganti, "A tractable approach to coverage and rate in cellular networks," IEEE Transactions on Communications, vol. 59, no. 11, pp. 3122-3134, November 2011.

[10] T. D. Novlan, H. S. Dhillon, and J. G. Andrews, "Analytical modeling of uplink cellular networks," IEEE Transactions on Wireless Communications, vol. 12, no. 6, pp. 2669-2679, June 2013.

[11] M. Peng, Y. Li, T. Q. S. Quek, and C. Wang, "Device-to-device underlaid cellular networks under rician fading channels," Wireless Communications, IEEE Transactions on, vol. 13, no. 8, pp. 42474259, 2014

[12] G. George, R. K. Mungara, and A. Lozano, "An analytical framework for device-to-device communication in cellular networks," IEEE Transactions on Wireless Communications, vol. 14, no. 11, pp. 6297 6310, Nov 2015.

[13] M. Ding and M. D. Lopez-Perez, "A new capacity scaling law in ultra-dense networks," arXiv:1704.00399 [cs.NI], Apr. 2017. [Online]. Available: https://arxiv.org/abs/1704.00399

[14] 3GPP, “TR 36.828 (v11.0.0): Further enhancements to LTE time division duplex (TDD) for downlink-uplink (DL-UL) interference management and traffic adaptation," Jun 2012.

[15] M. Ding, D. López-Pérez, G. Mao, P. Wang, and Z. Lin, "Will the area spectral efficiency monotonically grow as small cells go dense?' IEEE GLOBECOM 2015, pp. 1-7, Dec. 2015.

[16] M. Haenggi, "On distances in uniformly random networks," IEEE Transactions on Information Theory, vol. 51, no. 10, pp. 3584-3586, Oct 2005. 